\title{
Field resistance of Spodoptera litura (Lepidoptera: Noctuidae) to organophosphates, pyrethroids, carbamates and four newer chemistry insecticides in Hunan, China
}

\author{
Hong Tong $\cdot$ Qi Su $\cdot$ Xiaomao Zhou \\ Lianyang Bai
}

Received: 29 January 2013/Accepted: 25 April 2013/Published online: 7 May 2013

(c) The Author(s) 2013. This article is published with open access at Springerlink.com

\begin{abstract}
The present studies were carried out to evaluate resistance in the populations of Spodoptera litura Fab. (Lepidoptera, Noctuidae) from five districts of Hunan Province in China to various insecticides from 2010 to 2012 using a standard leaf dip bioassay method. For organophosphates and pyrethroids, resistance ratios compared with a susceptible Lab-BJ strain were in the range of 14-229-fold for organophosphates and 12-227-fold for pyrethroids. Similarly, relative low levels of resistance to emamectin, indoxacarb, and chlorfenapyr were observed in all five populations. In contrast, the resistance to carbamates (thiodicarb or methomyl) was significantly higher than that of organophosphates, pyrethroids and newer chemistry insecticides. The pairwise correlation coefficients of $\mathrm{LC}_{50}$ values indicated that the newer chemistry insecticides and old generation insecticides were not significant except abamectin, which was negatively significantly correlated with methomyl. A significant correlation was observed between thiodicarb, methomyl, and
\end{abstract}

Communicated by E. Roditakis.

H. Tong $\cdot$ Q. Su $\cdot$ X. Zhou $\cdot$ L. Bai $(\bowtie)$

Institute of Pesticide Science, Hunan Agricultural University,

Changsha 410128, People's Republic of China

e-mail: bailianyang2005@yahoo.com.cn

H. Tong

e-mail: tonghong1205@163.com

Q. Su

e-mail: suqicaas@163.com

X. Zhou

e-mail: Zhouxm1972@126.com

L. Bai

Hunan Institute of Humanities, Science and Technology, Loudi

417000, People's Republic of China deltamethrin, whereas resistance to bifenthrin showed no correlations with resistance to other insecticides except deltamethrin. The results are discussed in relation to integrated pest management for S. litura with special reference to management of field evolved resistance to insecticides.

Keywords Cutworm - Emamectin benzoate .

Abamectin · Indoxacarb · Chlorfenapyr .

Resistance evolution · Cross-resistance

\section{Introduction}

The cutworm Spodoptera litura Fab. (Lepidoptera, Noctuidae) is well known as a serious cosmopolitan pest with extensive host range of economically important crops such as cotton, groundnut, soybean, tomato, sweet potato, and many other crops (Matsuura and Naito 1997; Sahayaraj and Paulraj 1998). S. litura has been shown to be resistant to a wide range of insecticides, which has led to sporadic out breaks of the pest and failure of crops (Ahmad et al. 2007a). Since the pest was found in Hunan Province of China, its damage has increased continually. Its control has depended mostly on application of various insecticides. As a result, many field populations of this pest have developed high resistance against wide variety of insecticides including organophosphate, carbamate, pyrethroids and some selected newer chemistry insecticides with field control failure observed very frequently (Armes et al. 1997; Kranthi et al. 2001; Ahmad et al. 2007a, b, 2008; Saleem et al. 2008). The management of the pest has therefore become increasingly difficult all over the world and the most commonly used insecticides are ineffective in controlling it. 
Resistance to insecticides is a major problem associated with the chemical control of insect pests, which is characterized by rapid evolution under strong selection of gene(s) that confers survival to insecticides (Ahmad et al. 2008). This selective pressure exerted by the insecticides abruptly increases the frequency of the genetic condition expressed as resistance within the exposed population. The development of resistance is a result of the selection pressure exerted on sprayed populations increasing the frequency of resistant individuals (Torres-Vila et al. 2002), but several factors including temperature are also involved in influencing the evolution of insecticides resistance (Raymond and Marquine 1994). At present, the extensive use of conventional insecticides such as organophosphate, carbamate and pyrethroids against $S$. litura has produced prevalent resistance in China (Wu et al. 1995; Huang et al. 2006). With high resistance to conventional insecticides, the insect growth regulators (IGRs) and newer insecticides were recently introduced to control this pest (Chen et al. 2008; Su et al. 2012). In the case of IGRs, flufenoxuron, chlorfluazuron, tebufenozide, and methoxyfenozide were used to control S. litura in Shandong and Jiangsu Provinces and had high toxicity to $S$. litura, in which resistance to flufenoxuron and methoxyfenozide was barely produced (Huang et al. 2006). In addition, the newer insecticides bearing novel modes of action such as indoxacarb, abamectin, emamectin benzoate, fipronil, and spinosad were recently introduced into Hunan Province for management of the pests. The extensive use of these newer insecticides against $S$. litura have provided an ideal environment for its evolution of resistance and $S$. litura was found to have inherent risks for resistance to indoxacarb (Wang et al. 2009). Previous exposure and selection with insecticides can confer resistance to newly introduced insecticides through crossresistance (Bisset et al. 1997; Sayyed et al. 2008), reducing the effectiveness of many new insecticides. There are some data available on the newer insecticide resistance in $S$. litura from cash crops and vegetables growing countries such as Pakistan (Ahmad et al. 2008; Shad et al. 2012).

Following reports of poor efficacy of the newer chemistry insecticides against $S$. litura both in cultivated crops and vegetables and to supply accurate information for management of resistance and prevent its outbreak in the future, we surveyed resistance to the newer chemistry compounds, as well as conventional insecticides against $S$. litura from various zones of the Hunan Province of China to ascertain whether or not the resistance was indeed evolving. This study is expected to be helpful in devising management strategies to overcome the resistance problems and to control $S$. litura under field conditions in the future.

\section{Materials and methods}

Insects

A laboratory susceptible strain of S. litura was obtained from the Institute of Zoology, Beijing, China and designated as Lab-BJ. This strain was obtained by single pair crosses of a field-collected population of $S$. litura and reared in the laboratory for 6 years without exposure to insecticides. Bioassays were conducted in the laboratory to get the mortality data to use as a reference for baseline susceptibility of insecticides. Different populations of $S$. litura at fourth- or fifth-instar larvaes were collected from the field crops grown within a radius of almost $200 \mathrm{~km}$ of Hunan Province from 2010 to 2012. All strains were collected by walking through a 3 ha block of a particular crop in a zig-zag manner to get a mixed population from various areas (Fig. 1) and brought to the laboratory. The larvae were reared on semi-synthetic diet (Ahmad et al. 2008; Saleem et al. 2008) in the laboratory at $25 \pm 3{ }^{\circ} \mathrm{C}$ and $65 \pm 5 \% \mathrm{RH}$ in glass jars for at least two generations before the bioassays were carried out. Diet was replaced after $24 \mathrm{~h}$ and pupae were collected on alternate days. Moths were shifted to glass cages with mesh sides for ventilation and fed on a solution containing $10 \%$ honey soaked onto cotton wool ball (Ahmad et al. 2007b). The neonate larvae were fed with semi-synthetic diet. The fieldcollected populations were reared in the laboratory to accommodate to laboratory conditions and to obtain sufficient insect numbers for bioassays.

\section{Insecticides}

Ten insecticides were used in present study: $90 \%$ emamectin benzoate (Hebei Veyong Bio-Chemical Co., Ltd., Hebei, China); $97.2 \%$ abamectin (Hebei Veyong BioChemical Co., Ltd., Hebei, China); 97 \% indoxacarb (E.I. DuPont de Nemours and Co., Inc., Wilmington, DE, USA); 94.5\% chlorfenapyr (BASF (China) Co., Ltd., Beijing, China); $97.5 \%$ chlorpyrifos (Jinan Luba Chemicals Co., Ltd., Jinan City, China); $91.6 \%$ profenofos (Jiangsu Baoling Chemical Co., Ltd., Jiangsu, China); $95 \%$ thiodicarb (Bayer CropScience Hangzhou Co., Ltd., Hangzhou, China); $98 \%$ methomyl (Shandong Huayang Technology Co., Ltd., Ningyang, China); $98 \%$ deltamethrin (Nanjing Redsun Co., Ltd., Nanjing, China); and $95 \%$ bifenthrin (Bayer CropScience Hangzhou Co., Ltd., Hangzhou, China).

Bioassays

Bioassays were conducted with newly third-instar larvae of S. litura using a standard leaf disk method (Sayyed et al. 
Fig. 1 Sampling sites of Spodoptera litura in various zones of Hunan Province of China. The survey was carried out in the field season of 2010-2012. Surveyed province is highlighted in a green shad. (Color figure online)

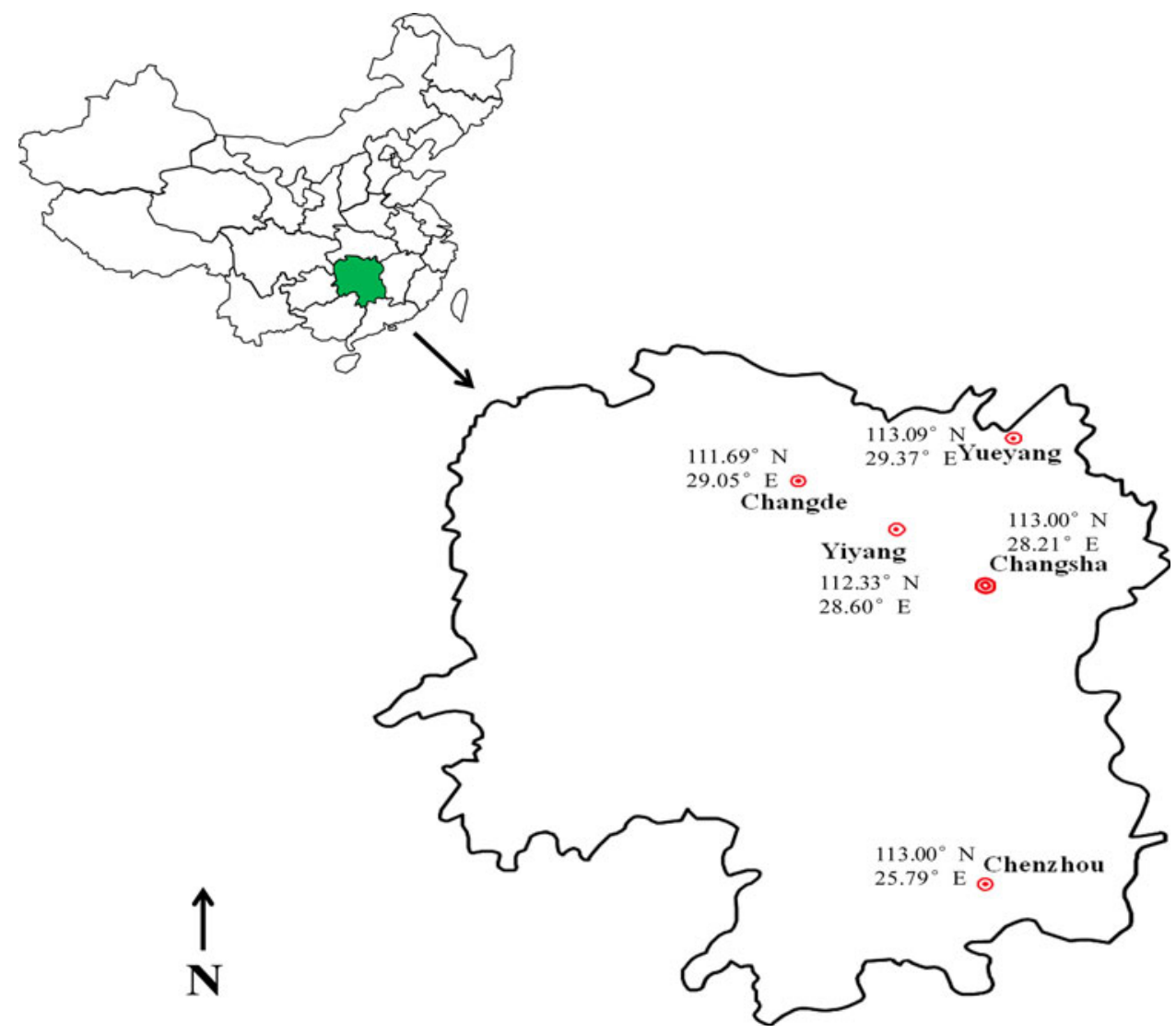

2000; Ahmad et al. 2007b). Serial dilutions as $\mathrm{mg} / \mathrm{l}$ of the active ingredient of the test compounds were prepared using $0.1 \%$ triton X-100 in water. Cotton leaves were cut into small, $9 \mathrm{~cm}$ pieces and dipped into the insecticide solution for $10 \mathrm{~s}$. These leaves were air dried at ambient temperature for 5-10 min by spreading on a towel paper. Leaves were dipped in sterile distilled water and $0.1 \%$ triton X-100 only to use as controls. Leaves treated with insecticides were then transferred to each Petri dish lined with moistened filter paper. At least six concentrations and four replications (10 larvae per replication) were used to estimate each concentration mortality line thus total numbers of tested larvae per concentration were 40 . The bioassays were kept at a temperature of $25 \pm 3{ }^{\circ} \mathrm{C}, 65 \pm 5 \%$ relative humidity, and photoperiod of 16:8 (light: dark). Mortality data were scored $48 \mathrm{~h}$ after exposure for insecticides. Larvaes were considered dead if they failed to make a coordinated movement when prodded with a brush.

Data analysis

Data obtained were corrected for control mortality using Abbott's formula (1925) where necessary, and were analyzed by probit analysis through POLO-Plus (LeOra 2003) to estimate $\mathrm{LC}_{50}$ values and their $95 \%$ fiducial limits (FLs). Resistance ratios (RRs) were determined as $\mathrm{LC}_{50}$ values of field strain/ $\mathrm{LC}_{50}$ values of $\mathrm{Lab}-\mathrm{BJ}$. $\mathrm{LC}_{50}$ values were considered significantly different when they did not overlap with each other at their respective $95 \%$ fiducial values (Litchfield and Wilcoxon 1949). The slope for regression line was compared with $t$ test using SPSS software. A cross-resistance mechanism was determined among the tested insecticides by pairwise correlation coefficients of $\log \mathrm{LC}_{50}$ values of the field populations by the Pearson correlation with the help of computer program XL-Stats.

\section{Results}

Toxicity of insecticides to laboratory strain

The results of bioassays for organophosphates against LabBJ showed that the profenofos was similar to the toxicity of chlorpyrifos based on the presence of overlap in the $95 \%$ FLs, and among the carbamates tested, thiodicarb was less toxic than methomyl $(P<0.05)$, and bifenthrin was more toxic than deltamethrin in pyrethroids tested $(P<0.05)$ (Table 1). Among the newer chemistry insecticides tested, the most toxic was indoxacarb, and emamectin benzoate was the least toxic among the tested insecticides against the laboratory strain of S. litura (Table 1). 
Table 1 Baseline susceptibilities of laboratory populations to ten insecticides

\begin{tabular}{llll}
\hline Insecticides & Toxic regression equation & $\mathrm{LC}_{50}(\mathrm{mg} / \mathrm{l})(95 \%$ FL) & Correlation coefficient \\
\hline Chlorpyrifos & $Y=3.965+1.671 X$ & $4.18(2.84-5.66)$ & 0.9904 \\
Profenofos & $Y=4.079+2.164 X$ & $3.75(2.99-4.51)$ & 0.9980 \\
Thiodicarb & $Y=3.609+2.681 X$ & $6.42(4.28-8.56)$ & 0.9925 \\
Methomyl & $Y=2.793+2.965 X$ & $1.28(0.72-2.29)$ & 0.9972 \\
Deltamethrin & $Y=1.964+3.722 X$ & $3.99(2.29-6.95)$ & 0.9948 \\
Bifenthrin & $Y=2.442+3.581 X$ & $0.51(0.24-1.07)$ & 0.9967 \\
Emamectin & $Y=1.307+4.716 X$ & $0.67(0.12-1.22)$ & 0.9942 \\
Abamectin & $Y=6.196+2.254 X$ & $0.28(0.15-0.46)$ & 0.9964 \\
Indoxacarb & $Y=2.814+3.798 X$ & $0.08(0.05-0.11)$ & 0.9992 \\
Chlorfenapyr & $Y=1.315+4.159 X$ & $0.54(0.37-0.71)$ & 0.9973 \\
\hline
\end{tabular}

Toxicity of conventional insecticides to field populations

In general, the RR for organophosphates ranged from 14to 229-fold compared with the Lab-BJ population. The resistance to chlorpyrifos in $S$. litura was the lowest in a population collected from Changde, while the highest resistance was obtained in a population collected from Yiyang (Table 2). The resistance to chlorpyrifos ranged from 22-fold in the Changsha population to 120 -fold in the Yiyang population in 2012. In the case of profenofos, $S$. litura in all five regions revealed higher resistance compared with chlorpyrifos tested, ranging from 24- (Changsha in 2010) to 229-fold (Changde in 2012) (Table 2). The average slope for the most of the field populations to organophosphates group was similar to the average slope of Lab-BJ population (Table 2).

Two carbamates, thiodicarb, and methomyl tested in the present study, had very high levels of resistance, ranging from 38- to 1,069-fold compared with Lab-BJ strain. The resistance to methomyl was the highest in the population collected from Yiyang in 2012 with a RR of 1,069-fold while the lowest resistance (38-fold) from Chenzhou in 2012 (Table 2). On average, the RR was significantly higher in the populations collected from cotton, soybean, and lotus than the populations from taro and sweet potato (Table 2).

Among the pyrethroids, the RR ranged from 12- to 227 -fold compared with the Lab-BJ population. The resistance to deltamethrin against $S$. litura was the lowest in a population collected from Yiyang in 2010, while the highest resistance to bifenthrin was obtained in a population collected from Changsha in 2012 (Table 2). In general, the average resistance level to deltamethrin and bifenthrin groups tested was equivalent. The average slope for the most of the field populations to pyrethroids group was similar to the average slope of Lab-BJ population; however, a substantial inter-population variation in slope was evident for bifenthrin, for example (3.43) for Changde population collected from cotton in 2012 (Table 2).

Toxicity of newer chemistry insecticides to field populations

Results of the toxicity of newer chemistry insecticides i.e., emamectin benzoate, abamectin, indoxacarb, and chlorfenapyr against different populations are shown in Table 3. Emamectin benzoate is a synthetic analog of abamectin and the RR ranged from 1- to 22-fold when compared with the Lab-BJ strain. Almost all the populations collected from five districts for 3 years were significant resistance compared with Lab-BJ strain. The resistance to emamectin from Changsha, Yiyang, and Chenzhou populations showed increasing levels of resistance from 2010 to 2012 .

Resistance level for abamectin ranged from 3- to 43-fold more than Lab-BJ strain. Populations collected from four locations, Changsha, Yueyang, Yiyang, and Chenzhou showed increasing trends in resistance levels, while Changde population in 2010-2012 showed varying levels of resistance. The population in 2012 from Chenzhou showed highest level of resistance with ratio of 43 -fold compared to Lab-BJ, whereas the lowest level of tolerance was observed from Changsha district in 2010 with RR of threefold compared to Lab-BJ.

Out of 14 field populations tested for indoxacarb, 3 populations showed moderate level of resistance (21-22fold), while other populations showed low level with a RR in the range of 2-17-fold was observed. The highest level of RR (22-fold) compared with Lab-BJ was observed from Changde in 2011 and Yueyang in 2012, whereas the lowest level of resistance (twofold) was recorded in a population from Yiyang (Table 3).

Spodoptera litura had exhibited low resistance to chlorfenapyr in general, with RRs commonly less than 20-fold compared with Lab-BJ (Table 3).The average 
Table 2 Toxicity of some organophosphates and synthetic pyrethroids insecticides against different populations of Spodoptera litura from Hunan, China

\begin{tabular}{|c|c|c|c|c|c|c|c|c|c|c|}
\hline \multirow[t]{2}{*}{ Insecticides } & \multirow[t]{2}{*}{ Location } & \multirow{2}{*}{$\begin{array}{l}\text { Collected } \\
\text { from }\end{array}$} & \multirow{2}{*}{$\begin{array}{l}\text { Collection } \\
\text { data }\end{array}$} & \multirow{2}{*}{$\begin{array}{l}\text { Dose range } \\
(\mathrm{mg} / \mathrm{l})\end{array}$} & \multirow[t]{2}{*}{$\mathrm{LC}_{50}(\mathrm{mg} / \mathrm{l})(95 \% \mathrm{FL})$} & \multirow[t]{2}{*}{ RR } & \multicolumn{3}{|c|}{ Fit of probit line } & \multirow[t]{2}{*}{$n$} \\
\hline & & & & & & & Slope $( \pm \mathrm{SE})$ & $\chi^{2}$ & df & \\
\hline \multirow[t]{14}{*}{ Chlorpyrifos } & \multirow[t]{3}{*}{ Changsha } & Taro & Sep-10 & $20-240$ & $82.13(42.37-120.23)$ & 19.7 & $1.44 \pm 0.32$ & 1.26 & 4 & 240 \\
\hline & & Cotton & Sep-11 & $25-300$ & $103.37(88.29-116.45)$ & 24.7 & $1.61 \pm 0.28$ & 4.00 & 4 & 240 \\
\hline & & Cotton & Oct-12 & $25-320$ & $91.71(46.37-137.02)$ & 21.9 & $1.53 \pm 0.22$ & 1.76 & 4 & 240 \\
\hline & \multirow[t]{3}{*}{ Yueyang } & Lotus & Sep-10 & $75-800$ & $307.06(162.12-450.38)$ & 73.5 & $1.26 \pm 0.47$ & 0.57 & 4 & 240 \\
\hline & & Cotton & Aug-11 & $50-600$ & $210.92(138.38-292.37)$ & 50.5 & $1.63 \pm 0.24$ & 0.39 & 4 & 240 \\
\hline & & Lotus & July-12 & $65-800$ & $262.55(216.77-306.86)$ & 62.8 & $1.72 \pm 0.34$ & 0.82 & 4 & 240 \\
\hline & \multirow[t]{3}{*}{ Changde } & Soybean & Aug-10 & $12-200$ & $56.51(30.27-82.76)$ & 13.5 & $1.26 \pm 0.17$ & 1.14 & 4 & 240 \\
\hline & & Cotton & Aug-11 & $30-480$ & $176.81(90.12-263.11)$ & 42.3 & $0.67 \pm 0.24$ & 0.65 & 4 & 240 \\
\hline & & Cotton & Oct-12 & $35-560$ & $198.13(95.4-296.86)$ & 47.4 & $1.19 \pm 0.21$ & 2.74 & 4 & 240 \\
\hline & \multirow[t]{3}{*}{ Yiyang } & Soybean & Oct-10 & $50-800$ & $230.32(140.12-320.24)$ & 55.1 & $1.31 \pm 0.24$ & 0.38 & 4 & 240 \\
\hline & & Cotton & July-11 & $50-600$ & $216.19(110.12-322.23)$ & 51.7 & $1.73 \pm 0.25$ & 0.41 & 4 & 240 \\
\hline & & Cotton & Aug-12 & $100-1000$ & $502.60(366.65-636.17)$ & 120 & $1.21 \pm 0.61$ & 0.26 & 4 & 240 \\
\hline & \multirow[t]{2}{*}{ Chenzhou } & Cotton & Oct-11 & $75-800$ & $335.36(263.47-405.36)$ & 80.2 & $1.38 \pm 0.25$ & 0.38 & 4 & 240 \\
\hline & & Sweet potato & Sep-12 & $75-800$ & $285.62(202.12-369.34)$ & 68.3 & $1.14 \pm 0.29$ & 0.81 & 4 & 240 \\
\hline \multirow[t]{14}{*}{ Profenofos } & \multirow[t]{3}{*}{ Changsha } & Taro & Sep-10 & $25-300$ & $90.6(61.20-119.37)$ & 24.2 & $1.48 \pm 0.25$ & 0.59 & 4 & 240 \\
\hline & & Cotton & Sep-11 & $50-600$ & $215.55(153.75-275.55)$ & 57.5 & $1.73 \pm 0.29$ & 3.04 & 4 & 240 \\
\hline & & Cotton & Oct-12 & $60-900$ & $276.86(157.84-397.35)$ & 73.8 & $1.67 \pm 0.43$ & 0.058 & 4 & 240 \\
\hline & \multirow[t]{3}{*}{ Yueyang } & Lotus & Sep-10 & $60-800$ & $248.36(142.33-354.68)$ & 66.2 & $1.46 \pm 0.36$ & 0.68 & 4 & 240 \\
\hline & & Cotton & Aug-11 & $75-900$ & $392.81(260.12-527.25)$ & 105 & $2.01 \pm 0.39$ & 0.73 & 4 & 240 \\
\hline & & Lotus & July-12 & $75-900$ & $323.89(200.23-446.68)$ & 86.4 & $0.82 \pm 0.26$ & 0.38 & 4 & 240 \\
\hline & \multirow[t]{3}{*}{ Changde } & Soybean & Aug-10 & $70-1120$ & $280.8(176.59-384.58)$ & 74.9 & $0.75 \pm 0.27$ & 1.59 & 4 & 240 \\
\hline & & Cotton & Aug-11 & $80-1280$ & $358.2(213.47-500.96)$ & 95.5 & $1.25 \pm 0.17$ & 1.34 & 4 & 240 \\
\hline & & Cotton & Oct-12 & $150-2400$ & 859.91 (644.87-1072.96) & 229 & $1.90 \pm 0.40$ & 1.47 & 4 & 240 \\
\hline & \multirow[t]{3}{*}{ Yiyang } & Soybean & Oct-10 & $35-560$ & $175.31(112.13-237.43)$ & 46.8 & $1.71 \pm 0.14$ & 0.29 & 4 & 240 \\
\hline & & Cotton & July-11 & $45-720$ & $198.15(120.17-276.13)$ & 52.8 & $0.92 \pm 0.36$ & 1.53 & 4 & 240 \\
\hline & & Cotton & Aug-12 & $35-560$ & $127.31(64.50-192.13)$ & 34.0 & $1.80 \pm 0.27$ & 0.81 & 4 & 240 \\
\hline & \multirow[t]{2}{*}{ Chenzhou } & Cotton & Oct-11 & $120-2000$ & $462.98(368.93-557.02)$ & 123 & $1.57 \pm 0.22$ & 2.16 & 4 & 240 \\
\hline & & Sweet potato & Sep-12 & $150-2400$ & $631.65(308.23-955.12)$ & 168 & $1.84 \pm 0.32$ & 0.66 & 4 & 240 \\
\hline \multirow[t]{14}{*}{ Thiodicarb } & Changsha & Taro & Sep-10 & $62.5-1000$ & $247.56(140.21-354.63)$ & 38.6 & $1.31 \pm 0.23$ & 1.69 & 4 & 240 \\
\hline & & Cotton & Sep-11 & $375-6000$ & $1762.87(825.35-2769.39)$ & 275 & $2.08 \pm 0.39$ & 0.80 & 4 & 240 \\
\hline & & Cotton & Oct-12 & $375-6000$ & $1657.13(1210.28-2101.22)$ & 258 & $2.02 \pm 0.27$ & 0.77 & 4 & 240 \\
\hline & Yueyang & Lotus & Sep-10 & $175-2800$ & 734.38 (410.35-1057.44) & 114 & $1.34 \pm 0.15$ & 1.83 & 3 & 240 \\
\hline & & Cotton & Aug-11 & $125-2000$ & $541.01(253.83-826.20)$ & 84.3 & $2.05 \pm 0.24$ & 0.17 & 4 & 240 \\
\hline & & Lotus & July-12 & $200-3200$ & $824.20(623.18-1024.38)$ & 128 & $2.11 \pm 0.36$ & 3.82 & 4 & 240 \\
\hline & Changde & Soybean & Aug-10 & $250-4000$ & $1177.88(732.16-1613.59)$ & 183 & $2.60 \pm 0.30$ & 2.78 & 4 & 240 \\
\hline & & Cotton & Aug-11 & $375-6000$ & $1486.62(731.92-2239.31)$ & 232 & $1.53 \pm 0.25$ & 3.30 & 4 & 240 \\
\hline & & Cotton & Oct-12 & $375-6000$ & $1620.02(1010.33-2228.56)$ & 252 & $1.71 \pm 0.24$ & 0.79 & 4 & 240 \\
\hline & Yiyang & Soybean & Oct-10 & $350-5600$ & 1404.18 (932.11-1873.25) & 219 & $0.89 \pm 0.26$ & 0.60 & 4 & 240 \\
\hline & & Cotton & July-11 & $450-7200$ & $1786.94(976.83-2583.06)$ & 278 & $1.31 \pm 0.24$ & 1.98 & 4 & 240 \\
\hline & & Cotton & Aug-12 & $625-10000$ & 2725.87 (1214.17-4237.57) & 425 & $2.47 \pm 0.48$ & 1.27 & 4 & 240 \\
\hline & Chenzhou & Cotton & Oct-11 & $175-2800$ & $707.81(342.37-1071.25)$ & 110 & $1.78 \pm 0.36$ & 0.84 & 4 & 240 \\
\hline & & Sweet potato & Sep-12 & $225-3600$ & $952.54(500.52-1406.13)$ & 148 & $1.51 \pm 0.66$ & 2.45 & 4 & 240 \\
\hline
\end{tabular}


Table 2 continued

\begin{tabular}{|c|c|c|c|c|c|c|c|c|c|c|}
\hline \multirow[t]{2}{*}{ Insecticides } & \multirow[t]{2}{*}{ Location } & \multirow{2}{*}{$\begin{array}{l}\text { Collected } \\
\text { from }\end{array}$} & \multirow{2}{*}{$\begin{array}{l}\text { Collection } \\
\text { data }\end{array}$} & \multirow{2}{*}{$\begin{array}{l}\text { Dose range } \\
(\mathrm{mg} / \mathrm{l})\end{array}$} & \multirow[t]{2}{*}{$\mathrm{LC}_{50}(\mathrm{mg} / \mathrm{l})(95 \% \mathrm{FL})$} & \multirow[t]{2}{*}{$\mathrm{RR}$} & \multicolumn{3}{|c|}{ Fit of probit line } & \multirow[t]{2}{*}{$n$} \\
\hline & & & & & & & Slope $( \pm$ SE $)$ & $\chi^{2}$ & $\mathrm{df}$ & \\
\hline \multirow[t]{14}{*}{ Methomyl } & \multirow[t]{3}{*}{ Changsha } & Taro & Sep-10 & $15-240$ & $59.84(34.17-85.51)$ & 46.8 & $2.14 \pm 0.24$ & 0.99 & 4 & 240 \\
\hline & & Cotton & Sep-11 & $150-2400$ & $624.41(420.24-826.33)$ & 488 & $1.47 \pm 0.55$ & 0.39 & 4 & 240 \\
\hline & & Cotton & Oct-12 & $200-3200$ & $786.21(317.26-1254.25)$ & 614 & $1.37 \pm 0.36$ & 1.34 & 4 & 240 \\
\hline & \multirow[t]{3}{*}{ Yueyang } & Lotus & Sep-10 & $30-480$ & $118.23(72.35-164.12)$ & 92.4 & $1.81 \pm 0.18$ & 0.76 & 4 & 240 \\
\hline & & Cotton & Aug-11 & $70-1120$ & $285.93(180.72-389.93)$ & 223 & $1.84 \pm 0.19$ & 1.50 & 4 & 240 \\
\hline & & Lotus & July-12 & $62.5-1000$ & $247.26(124.38-489.75)$ & 193 & $1.63 \pm 0.28$ & 0.28 & 4 & 240 \\
\hline & \multirow[t]{3}{*}{ Changde } & Soybean & Aug-10 & $50-800$ & $215.76(145.58-285.27)$ & 169 & $2.01 \pm 0.25$ & 1.16 & 4 & 240 \\
\hline & & Cotton & Aug-11 & $87.5-1400$ & $350.95(240.37-460.57)$ & 274 & $2.22 \pm 0.21$ & 0.73 & 4 & 240 \\
\hline & & Cotton & Oct-12 & $100-1600$ & $400.96(318.37-504.74)$ & 313 & $2.26 \pm 0.51$ & 1.18 & 4 & 240 \\
\hline & \multirow[t]{3}{*}{ Yiyang } & Soybean & Oct-10 & $125-2000$ & $469.44(280.17-658.71)$ & 367 & $2.15 \pm 0.60$ & 1.57 & 4 & 240 \\
\hline & & Cotton & July-11 & $250-3000$ & $1055.53(738.74-1372.32)$ & 825 & $1.73 \pm 0.18$ & 0.18 & 4 & 240 \\
\hline & & Cotton & Aug-12 & $325-4000$ & $1368.59(628.31-2107.71)$ & 1,069 & $2.23 \pm 0.53$ & 2.60 & 4 & 240 \\
\hline & \multirow[t]{2}{*}{ Chenzhou } & Cotton & Oct-11 & $20-320$ & $87.40(72.30-100.49)$ & 68.3 & $1.94 \pm 0.24$ & 0.80 & 4 & 240 \\
\hline & & Sweet potato & Sep-12 & $12.5-200$ & $47.97(23.25-72.70)$ & 37.5 & $2.02 \pm 0.45$ & 0.74 & 4 & 240 \\
\hline \multirow[t]{14}{*}{ Deltamethrin } & \multirow[t]{3}{*}{ Changsha } & Taro & Sep-10 & $25-400$ & $131.15(93.57-168.73)$ & 32.9 & $1.59 \pm 0.18$ & 0.42 & 4 & 240 \\
\hline & & Cotton & Sep-11 & $112.5-1800$ & $460.96(300.26-620.87)$ & 116 & $1.88 \pm 0.21$ & 1.33 & 4 & 240 \\
\hline & & Cotton & Oct-12 & $37.5-2200$ & $562.91(304.55-1040.43)$ & 141 & $1.53 \pm 0.35$ & 0.21 & 4 & 240 \\
\hline & \multirow[t]{3}{*}{ Yueyang } & Lotus & Sep-10 & $17.5-280$ & $67.46(43.53-90.60)$ & 16.9 & $1.80 \pm 0.37$ & 0.20 & 4 & 240 \\
\hline & & Cotton & Aug-11 & $25-400$ & $142.12(92.70-190.56)$ & 35.6 & $1.57 \pm 0.15$ & 0.49 & 4 & 240 \\
\hline & & Lotus & July-12 & $30-480$ & $113.24(72.13-154.34)$ & 28.4 & $0.92 \pm 0.28$ & 1.41 & 4 & 240 \\
\hline & \multirow[t]{3}{*}{ Changde } & Soybean & Aug-10 & $37.5-600$ & $156.21(97.34-215.08)$ & 39.2 & $1.91 \pm 0.45$ & 1.59 & 4 & 240 \\
\hline & & Cotton & Aug-11 & $87.5-1400$ & $367.60(220.38-512.82)$ & 92.1 & $0.82 \pm 0.13$ & 1.08 & 4 & 240 \\
\hline & & Cotton & Oct-12 & $150-2400$ & $622.74(329.41-1177.26)$ & 156 & $1.68 \pm 0.32$ & 0.83 & 4 & 240 \\
\hline & \multirow[t]{3}{*}{ Yiyang } & Soybean & Oct-10 & $17.5-280$ & 69.35 (40.37-98.32) & 17.4 & $1.13 \pm 0.12$ & 1.14 & 4 & 240 \\
\hline & & Cotton & July-11 & $75-1200$ & $333.05(212.57-453.52)$ & 83.5 & $0.92 \pm 0.24$ & 0.95 & 4 & 240 \\
\hline & & Cotton & Aug-12 & $175-2800$ & $693.52(315.79-1523.06)$ & 174 & $1.38 \pm 0.25$ & 1.61 & 4 & 240 \\
\hline & \multirow[t]{2}{*}{ Chenzhou } & Cotton & Oct-11 & $35-560$ & $137.58(76.39-197.46)$ & 34.5 & $1.57 \pm 0.15$ & 1.23 & 4 & 240 \\
\hline & & Sweet potato & Sep-12 & $75-1200$ & $329.73(152.63-505.77)$ & 82.6 & $1.47 \pm 0.42$ & 0.62 & 4 & 240 \\
\hline \multirow[t]{14}{*}{ Bifenthrin } & Changsha & Taro & Sep-10 & $2.5--30$ & $8.80(7.23-10.38)$ & 17.3 & $1.55 \pm 0.25$ & 3.32 & 4 & 240 \\
\hline & & Cotton & Sep-11 & $22.5-320$ & 89.80 (71.85-107.74) & 176 & $1.66 \pm 0.46$ & 1.98 & 4 & 240 \\
\hline & & Cotton & Oct-12 & $25-400$ & $116.02(57.80-175.21)$ & 227 & $1.73 \pm 0.26$ & 0.56 & 4 & 240 \\
\hline & Yueyang & Lotus & Sep-10 & $2-32$ & $7.61(6.25-8.98)$ & 14.9 & $1.13 \pm 0.29$ & 0.60 & 4 & 240 \\
\hline & & Cotton & Aug-11 & $7.5-120$ & $29.82(20.36-39.29)$ & 58.5 & $2.33 \pm 0.31$ & 3.57 & 4 & 240 \\
\hline & & Lotus & July-12 & $10-160$ & $35.70(19.82-64.30)$ & 70.0 & $1.85 \pm 0.22$ & 0.49 & 4 & 240 \\
\hline & Changde & Soybean & Aug-10 & $1.5-24$ & $6.28(4.30-8.26)$ & 12.3 & $1.99 \pm 0.19$ & 2.88 & 4 & 240 \\
\hline & & Cotton & Aug-11 & $7.5-120$ & $32.23(21.14-43.32)$ & 63.2 & $1.39 \pm 0.26$ & 1.08 & 4 & 240 \\
\hline & & Cotton & Oct-12 & $7.5-120$ & $28.46(16.75-48.35)$ & 55.8 & $3.43 \pm 0.38$ & 0.39 & 4 & 240 \\
\hline & Yiyang & Soybean & Oct-10 & $5-80$ & $22.88(16.59-29.17)$ & 44.9 & $2.04 \pm 0.20$ & 1.36 & 4 & 240 \\
\hline & & Cotton & July-11 & $5-80$ & $19.17(12.32-26.01)$ & 37.6 & $1.73 \pm 0.18$ & 0.92 & 4 & 240 \\
\hline & & Cotton & Aug-12 & $17.5-280$ & $71.80(42.80-120.45)$ & 141 & $2.03 \pm 0.37$ & 0.56 & 4 & 240 \\
\hline & Chenzhou & Cotton & Oct-11 & $12.5-200$ & $49.24(34.69-63.79)$ & 96.6 & $2.00 \pm 0.29$ & 1.76 & 4 & 240 \\
\hline & & Sweet potato & Sep-12 & $15-240$ & $62.40(30.35-94.45)$ & 122 & $1.76 \pm 0.42$ & 0.44 & 4 & 240 \\
\hline
\end{tabular}

$R R$ resistance ratio, calculated as $\mathrm{LC}_{50}$ of field/ $\mathrm{LC}_{50}$ of Lab-BJ, $n$ number of larvae used in bioassay, including controls, $\chi^{2}$ values were not significant for all the assays 
Table 3 Toxicity of some newer insecticides against different populations of Spodoptera litura from Hunan, China

\begin{tabular}{|c|c|c|c|c|c|c|c|c|c|c|}
\hline \multirow[t]{2}{*}{ Insecticides } & \multirow[t]{2}{*}{ Location } & \multirow{2}{*}{$\begin{array}{l}\text { Collected } \\
\text { from }\end{array}$} & \multirow{2}{*}{$\begin{array}{l}\text { Collection } \\
\text { data }\end{array}$} & \multirow{2}{*}{$\begin{array}{l}\text { Dose range } \\
(\mathrm{mg} / \mathrm{l})\end{array}$} & \multirow{2}{*}{$\begin{array}{l}\mathrm{LC}_{50}(\mathrm{mg} / \mathrm{l}) \\
(95 \% \mathrm{FL})\end{array}$} & \multirow[t]{2}{*}{$\mathrm{RR}$} & \multicolumn{3}{|c|}{ Fit of probit line } & \multirow[t]{2}{*}{$n$} \\
\hline & & & & & & & Slope $( \pm \mathrm{SE})$ & $\chi^{2}$ & $\mathrm{df}$ & \\
\hline \multirow[t]{14}{*}{ Emamectin benzoate } & \multirow[t]{3}{*}{ Changsha } & Taro & Sep-10 & $0.25-4$ & $0.97(0.15-1.79)$ & 1.45 & $1.60 \pm 0.19$ & 0.61 & 4 & 240 \\
\hline & & Cotton & Sep-11 & $0.5-8$ & $2.40(1.87-2.93)$ & 3.58 & $2.07 \pm 0.23$ & 1.59 & 4 & 240 \\
\hline & & Cotton & Oct-12 & $1.25-20$ & $6.95(4.83-9.07)$ & 10.4 & $1.91 \pm 0.22$ & 2.60 & 4 & 240 \\
\hline & \multirow[t]{3}{*}{ Yueyang } & Lotus & Sep-10 & $0.25-4$ & $1.51(1.13-1.89)$ & 2.25 & $2.18 \pm 0.40$ & 0.33 & 4 & 240 \\
\hline & & Cotton & Aug-11 & $0.5-8$ & $3.33(2.75-3.91)$ & 4.97 & $1.89 \pm 0.36$ & 0.85 & 4 & 240 \\
\hline & & Lotus & July-12 & $0.5-8$ & $3.24(2.58-3.89)$ & 4.83 & $1.58 \pm 0.18$ & 2.23 & 4 & 240 \\
\hline & \multirow[t]{3}{*}{ Changde } & Soybean & Aug-10 & $0.25-4$ & $1.86(1.55-2.18)$ & 2.78 & $1.67 \pm 0.19$ & 1.38 & 4 & 240 \\
\hline & & Cotton & Aug-11 & $1-16$ & $5.24(4.05-6.43)$ & 7.82 & $1.55 \pm 0.15$ & 1.33 & 4 & 240 \\
\hline & & Cotton & Oct-12 & $1-16$ & $4.90(3.67-6.22)$ & 7.31 & $1.63 \pm 0.28$ & 0.76 & 4 & 240 \\
\hline & \multirow[t]{3}{*}{ Yiyang } & Soybean & Oct-10 & $0.5-8$ & $3.44(3.12-3.75)$ & 5.13 & $2.02 \pm 0.20$ & 3.13 & 4 & 240 \\
\hline & & Cotton & July-11 & $2.5-40$ & $11.58(7.38-15.79)$ & 17.3 & $1.84 \pm 0.29$ & 0.72 & 4 & 240 \\
\hline & & Cotton & Aug-12 & $1.25-20$ & $6.29(5.40-7.19)$ & 22.5 & $1.35 \pm 0.33$ & 0.35 & 4 & 240 \\
\hline & \multirow[t]{2}{*}{ Chenzhou } & Cotton & Oct-11 & $1.25-20$ & $5.82(4.02-7.61)$ & 8.68 & $1.69 \pm 0.23$ & 0.80 & 4 & 240 \\
\hline & & Sweet potato & Sep-12 & $2-32$ & $8.15(3.93-12.36)$ & 12.2 & $1.37 \pm 0.35$ & 0.54 & 4 & 240 \\
\hline \multirow[t]{14}{*}{ Abamectin } & \multirow[t]{3}{*}{ Changsha } & Taro & Sep-10 & $0.15-2.4$ & $0.74(0.53-0.95)$ & 2.64 & $1.94 \pm 0.24$ & 2.01 & 4 & 240 \\
\hline & & Cotton & Sep-11 & $0.5-8$ & $2.42(1.75-3.14)$ & 8.64 & $1.80 \pm 0.35$ & 0.17 & 4 & 240 \\
\hline & & Cotton & Oct-12 & $1.25-20$ & $5.83(4.35-7.31)$ & 20.8 & $1.73 \pm 0.16$ & 0.79 & 4 & 240 \\
\hline & \multirow[t]{3}{*}{ Yueyang } & Lotus & Sep-10 & $1.875-30$ & $10.55(7.92-13.17)$ & 15.7 & $1.83 \pm 0.25$ & 1.88 & 4 & 240 \\
\hline & & Cotton & Aug-11 & $1.25-20$ & $4.41(3.29-5.52)$ & 19.4 & $1.53 \pm 0.34$ & 0.54 & 4 & 240 \\
\hline & & Lotus & July-12 & $1.875-30$ & $9.69(8.32-11.06)$ & 34.6 & $2.21 \pm 0.24$ & 0.38 & 4 & 240 \\
\hline & \multirow[t]{3}{*}{ Changde } & Soybean & Aug-10 & $0.625-10$ & $2.93(2.16-3.71)$ & 10.5 & $2.06 \pm 0.37$ & 2.22 & 4 & 240 \\
\hline & & Cotton & Aug-11 & $0.75-12$ & $4.34(3.13-5.56)$ & 15.5 & $1.94 \pm 0.28$ & 1.16 & 4 & 240 \\
\hline & & Cotton & Oct-12 & $0.75-12$ & $3.86(3.09-4.61)$ & 13.8 & $1.75 \pm 0.35$ & 0.49 & 4 & 240 \\
\hline & \multirow[t]{3}{*}{ Yiyang } & Soybean & Oct-10 & $0.25-4$ & $1.72(1.16-2.27)$ & 6.13 & $2.20 \pm 0.22$ & 0.72 & 4 & 240 \\
\hline & & Cotton & July-11 & $1-16$ & $5.00(3.75-6.24)$ & 17.8 & $2.00 \pm 0.26$ & 1.30 & 4 & 240 \\
\hline & & Cotton & Aug-12 & $1.25-20$ & $6.29(5.40-7.19)$ & 22.5 & $1.35 \pm 0.33$ & 0.35 & 4 & 240 \\
\hline & \multirow[t]{2}{*}{ Chenzhou } & Cotton & Oct-11 & $1-16$ & $5.07(3.37-6.76)$ & 18.1 & $1.69 \pm 0.33$ & 0.28 & 4 & 240 \\
\hline & & Sweet potato & Sep-12 & $2.5-40$ & $11.93(7.27-16.59)$ & 42.6 & $1.60 \pm 0.41$ & 0.59 & 4 & 240 \\
\hline \multirow[t]{14}{*}{ Indoxacarb } & Changsha & Taro & Sep-10 & $0.075-1.2$ & $0.33(0.26-0.41)$ & 4.18 & $1.72 \pm 0.27$ & 2.60 & 4 & 240 \\
\hline & & Cotton & Sep-11 & $0.2-3.2$ & $0.80(0.62-0.98)$ & 10.0 & $1.47 \pm 0.55$ & 0.99 & 4 & 240 \\
\hline & & Cotton & Oct-12 & $0.25-4$ & $1.01(0.72-1.30)$ & 12.6 & $1.35 \pm 0.33$ & 0.35 & 4 & 240 \\
\hline & Yueyang & Lotus & Sep-10 & $0.05-0.8$ & $0.23(0.18-0.29)$ & 2.92 & $1.75 \pm 0.37$ & 0.88 & 4 & 240 \\
\hline & & Cotton & Aug-11 & $0.25-4$ & $1.37(1.15-1.60)$ & 17.2 & $1.86 \pm 0.35$ & 1.66 & 4 & 240 \\
\hline & & Lotus & July-12 & $0.5-8$ & $1.77(1.11-2.47)$ & 22.1 & $1.51 \pm 0.29$ & 0.52 & 4 & 240 \\
\hline & Changde & Soybean & Aug-10 & $0.25-4$ & $1.10(0.88-1.31)$ & 13.7 & $1.81 \pm 0.29$ & 0.18 & 4 & 240 \\
\hline & & Cotton & Aug-11 & $0.5-8$ & $1.79(1.37-2.21)$ & 22.4 & $2.24 \pm 0.21$ & 1.16 & 4 & 240 \\
\hline & & Cotton & Oct-12 & $0.5-8$ & $1.64(0.98-2.38)$ & 20.5 & $1.73 \pm 0.35$ & 0.41 & 4 & 240 \\
\hline & Yiyang & Soybean & Oct-10 & $0.025-0.4$ & $0.15(0.12-0.19)$ & 1.92 & $1.95 \pm 0.38$ & 0.72 & 4 & 240 \\
\hline & & Cotton & July-11 & $0.25-4$ & $1.29(0.83-1.74)$ & 16.1 & $1.67 \pm 0.27$ & 1.58 & 4 & 240 \\
\hline & & Cotton & Aug-12 & $0.25-4$ & $1.15(0.51-1.79)$ & 14.4 & $1.58 \pm 0.34$ & 0.44 & 4 & 240 \\
\hline & Chenzhou & Cotton & Oct-11 & $0.05-0.8$ & $0.29(0.22-0.36)$ & 3.65 & $1.48 \pm 0.24$ & 1.38 & 4 & 240 \\
\hline & & Sweet potato & Sep-12 & $0.125-2$ & $0.62(0.311-0.921)$ & 7.70 & $1.61 \pm 0.37$ & 0.62 & 4 & 240 \\
\hline
\end{tabular}


Table 3 continued

\begin{tabular}{|c|c|c|c|c|c|c|c|c|c|c|}
\hline \multirow[t]{2}{*}{ Insecticides } & \multirow[t]{2}{*}{ Location } & \multirow{2}{*}{$\begin{array}{l}\text { Collected } \\
\text { from }\end{array}$} & \multirow{2}{*}{$\begin{array}{l}\text { Collection } \\
\text { data }\end{array}$} & \multirow{2}{*}{$\begin{array}{l}\text { Dose range } \\
(\mathrm{mg} / \mathrm{l})\end{array}$} & \multirow{2}{*}{$\begin{array}{l}\mathrm{LC}_{50}(\mathrm{mg} / \mathrm{l}) \\
(95 \% \mathrm{FL})\end{array}$} & \multirow[t]{2}{*}{$\mathrm{RR}$} & \multicolumn{3}{|c|}{ Fit of probit line } & \multirow[t]{2}{*}{$n$} \\
\hline & & & & & & & Slope $( \pm$ SE $)$ & $\chi^{2}$ & $\mathrm{df}$ & \\
\hline \multirow[t]{14}{*}{ Chlorfenapyr } & \multirow[t]{3}{*}{ Changsha } & Taro & Sep-10 & $0.5-8$ & $2.51(1.97-3.04)$ & 4.64 & $1.73 \pm 0.16$ & 2.01 & 4 & 240 \\
\hline & & Cotton & Sep-11 & $0.5-8$ & $2.10(1.46-2.73)$ & 3.88 & $1.57 \pm 0.32$ & 0.38 & 4 & 240 \\
\hline & & Cotton & Oct-12 & $1.25-20$ & $5.63(3.05-10.40)$ & 10.4 & $1.70 \pm 0.35$ & 0.11 & 4 & 240 \\
\hline & \multirow[t]{3}{*}{ Yueyang } & Lotus & Sep-10 & $0.625-10$ & $2.58(1.97-3.18)$ & 4.77 & $1.94 \pm 0.24$ & 0.32 & 4 & 240 \\
\hline & & Cotton & Aug-11 & $0.75-12$ & $4.13(3.16-5.09)$ & 7.64 & $2.10 \pm 0.33$ & 0.45 & 4 & 240 \\
\hline & & Lotus & July-12 & $0.75-12$ & $3.52(2.73-4.31)$ & 6.52 & $1.81 \pm 0.32$ & 0.07 & 4 & 240 \\
\hline & \multirow[t]{3}{*}{ Changde } & Soybean & Aug-10 & $1.5-24$ & $8.14(6.32-9.97)$ & 15.1 & $1.71 \pm 0.24$ & 1.71 & 4 & 240 \\
\hline & & Cotton & Aug-11 & $1.5-24$ & $9.72(8.43-11.01)$ & 18.0 & $1.36 \pm 0.23$ & 0.28 & 4 & 240 \\
\hline & & Cotton & Oct-12 & $1.5-24$ & $7.62(3.77-15.41)$ & 14.1 & $1.62 \pm 0.36$ & 0.40 & 4 & 240 \\
\hline & \multirow[t]{3}{*}{ Yiyang } & Soybean & Oct-10 & $1-16$ & $4.43(3.78-5.08)$ & 8.20 & $1.83 \pm 0.25$ & 2.04 & 4 & 240 \\
\hline & & Cotton & July-11 & $1.25-20$ & $5.05(3.92-6.18)$ & 9.35 & $1.61 \pm 0.56$ & 0.26 & 4 & 240 \\
\hline & & Cotton & Aug-12 & $2-32$ & $8.86(3.88-20.23)$ & 16.4 & $2.17 \pm 0.35$ & 0.48 & 4 & 240 \\
\hline & \multirow[t]{2}{*}{ Chenzhou } & Cotton & Oct-11 & $1-16$ & $4.41(3.35-5.46)$ & 8.16 & $1.78 \pm 0.18$ & 0.79 & 4 & 240 \\
\hline & & Sweet potato & Sep-12 & $0.7-12$ & $3.06(1.63-4.48)$ & 5.66 & $1.57 \pm 0.31$ & 1.01 & 4 & 240 \\
\hline
\end{tabular}

$R R$ resistance ratio, calculated as $\mathrm{LC}_{50}$ of field/LC $\mathrm{LC}_{50}$ of Lab-BJ, $n$ number of larvae used in bioassay, including controls, $\chi^{2}$ values was not significant for all the assays

slope for regression lines was similar for all five field populations except for Lab-BJ (Table 3).

Pairwise correlations between $\log \mathrm{LC}_{50}$ values of different insecticides

Correlation between the newer chemistry insecticides and old generation insecticides was not significant $(P<0.05)$ except abamectin, which was significant but negatively correlated with methomyl (Table 4). A significant correlation was observed between thiodicarb, methomyl, and deltamethrin $(P<0.01)$, whereas resistance to bifenthrin showed no correlations with resistance to other insecticides except deltamethrin $(P<0.05)$. There was lack of crossresistance for emamectin, abamectin, indoxacarb, chlorfenapyr, chlorpyrifos, and profenofos in populations of $S$. litura from Hunan.

\section{Discussion}

The present study, conducted from 2010 to 2012, demonstrate that the $S$. litura populations on five cash crops in five regions of Hunan Province have shown varying degrees of resistance to six conventional insecticides and four newer insecticides. This suggests that populations of $S$. litura have the potential to develop resistance to a wide range of chemicals.

The resistance to organophosphates, which act as acetylcholinesterase inhibitors (Ahmad et al. 2007a, b), was found at a high level ( $>50$-fold) in most of populations except the populations collected from Changsha and Changde, which was medium level (20-50-fold) resistance to chlorpyrifos (Table 2). This could be related to the commonly reliance in the use of organophosphates against insects in these areas. Resistance in S. litura against organophosphates has been reported from various parts of the Asia countries, such as Pakistan (Ahmad et al. 2007a, b; Saleem et al. 2008; Shad et al. 2012), India (Armes et al. 1997; Kranthi et al. 2002), and China (Huang et al. 2006). There were also reports of resistance development in beet armyworm Spodoptera exigua (H.), a species closely related to S. litura, from Guatemala (Delorme et al. 1988), Mexico (Teran-Vargas et al. 1997), Nicaragua (Pérez et al. 2000), Pakistan (Ahmad and Arif 2010; Ishtiaq et al. 2012), and China (Mu et al. 2005; Zhou et al. 2011), providing evidence of high level of resistance against organophosphates insecticides. As carbamates were more effective insecticide against lepidopteran pests, including Spodoptera spp. (Ahmad et al. 2008; Saleem et al. 2008; Shad et al. 2012), the application of this insecticide group was widely used to control $S$. litura in recent years. In most areas of Hunan, farmers used carbamates more than five times a month, so all the populations showed very high level of resistance except the population from taro, which showed moderate level of resistance against carbamates, as taro was sporadic cultivation and insecticide was seldom for use in such vegetable. The resistance to synthetic pyrethroids (deltamethrin and bifenthrin) was found at high or very high level in all populations collected from Hunan in 2012 except the deltamethrin population from Yueyang, as this insecticide was forbidden for use in vegetables for 
Table 4 Pairwise correlation coefficient comparison between $\log \mathrm{LC}_{50}$ values of tested insecticides on field populations of Spodoptera litura

\begin{tabular}{|c|c|c|c|c|c|c|c|c|c|}
\hline & Emamectin & Abamectin & Indoxacarb & Chlorfenapyr & Chlorpyrifos & Thiodicarb & Profenofos & Methomyl & Deltamethrin \\
\hline Abamectin & $0.280^{\mathrm{ns}}$ & & & & & & & & \\
\hline Indoxacarb & $0.240^{\mathrm{ns}}$ & $0.06^{\mathrm{ns}}$ & & & & & & & \\
\hline Chlorfenapyr & $0.235^{\mathrm{ns}}$ & $-0.194^{\mathrm{ns}}$ & $0.267^{\mathrm{ns}}$ & & & & & & \\
\hline Chlorpyrifos & $0.301^{\mathrm{ns}}$ & $0.516^{\mathrm{ns}}$ & $-0.070^{\mathrm{ns}}$ & $0.116^{\mathrm{ns}}$ & & & & & \\
\hline Thiodicarb & $0.460^{\mathrm{ns}}$ & $-0.083^{\mathrm{ns}}$ & $0.318^{\mathrm{ns}}$ & $0.374^{\mathrm{ns}}$ & $0.296^{\mathrm{ns}}$ & & & & \\
\hline Profenofos & $0.207^{\mathrm{ns}}$ & $0.287^{\mathrm{ns}}$ & $0.306^{\mathrm{ns}}$ & $0.155^{\mathrm{ns}}$ & $0.054^{\mathrm{ns}}$ & $-0.109^{\mathrm{ns}}$ & & & \\
\hline Methomyl & $0.517^{\mathrm{ns}}$ & $-0.373^{0.034}$ & $0.282^{\mathrm{ns}}$ & $0.400^{\mathrm{ns}}$ & $0.304^{\mathrm{ns}}$ & $0.289^{\mathrm{ns}}$ & $-0.357^{\mathrm{ns}}$ & & \\
\hline Deltamethrin & $0.446^{\mathrm{ns}}$ & $-0.022^{\mathrm{ns}}$ & $0.418^{\mathrm{ns}}$ & $0.498^{\mathrm{ns}}$ & $0.148^{\mathrm{ns}}$ & $0.804^{0.001}$ & $0.236^{\mathrm{ns}}$ & $0.683^{0.007}$ & \\
\hline Bifenthrin & $0.322^{\text {ns }}$ & $0.146^{\mathrm{ns}}$ & $0.047^{\mathrm{ns}}$ & $-0.017^{\mathrm{ns}}$ & $0.066^{\mathrm{ns}}$ & $0.474^{\mathrm{ns}}$ & $0.022^{\mathrm{ns}}$ & $0.432^{\mathrm{ns}}$ & $0.639^{0.014}$ \\
\hline
\end{tabular}

Superscripts represent significance of the regression

export in Yueyang. The tendency of increasing resistance to pyrethroids is consistent with the results of Huang et al. (2006) and Xie et al. (2010), and this could be related to the increase in the use of pyrethroids in these areas.

Although variation in susceptibility to laboratory strain was observed among the newer insecticides tested, the magnitude of the differences was small, less than ninefold for these four newer insecticides (Table 1). These results suggest that the observed susceptibility differences reflect natural variation in laboratory strain susceptibility among the newer insecticides rather than variation caused by prior exposure to selection pressure. Overall, the laboratory strain was relatively more sensitive than the field populations, particularly to indoxacarb (Table 1). Different members of newer chemistry insecticides exhibited different levels of toxicity, which will be helpful in devising management strategies. Emamectin benzoate and abamectin belong to the avermectins group and act as chloride channel activators (Teran-Vargas et al. 1997). Emamectin looked to be an effective insecticide because it exhibited low level of resistance in most of the populations tested. Therefore, emamectin is still considered as an effective tool for management of $S$. litura for most of the areas. Indoxacarb acts as a voltage-dependent sodium channel blocker belonging to the oxadiazine insecticide group (Sayyed et al. 2008), and chlorfenapyr has a novel mode of action, targeting oxidative pathways in insect mitochondria (Van Leeuwen et al. 2006). Indoxacarb and chlorfenapyr exhibited low level of resistance in all populations tested except only one medium resistance population, suggesting its effectiveness for $S$. litura management for most of the areas. The low application of newer insecticides is also associated with their high price, which many farmers could not afford. However, this cannot explain why abamectin resulted in higher resistance compared with other newer insecticides in most of the populations in 2012, and pairwise comparisons of the $\log \mathrm{LC}_{50}$ values of insecticides tested showed occurrence of correlation within abamectin and methomyl (Table 4), which suggest that resistance to abamectin might due to a possible cross-resistance mechanism to conventional insecticides. A significant higher correlation between abamectin and emamectin benzoate has been reported from $S$. litura in Pakistan (Ahmad et al. 2008), our papers do not derive this results, although abamectin and emamectin both bind to the GABA-gated chloride channel. Previous studies reported that the detoxification enhancement causes metabolism resistance and involves different enzymes, including cytochrome P450 monooxygenase (MFO), carboxylesterase and esterase (Ishaaya and Casida 1980; Scott 1999; Huang et al. 2006; Chen et al. 2008) and both MFO and esterase have many isoenzymes which all have a range of substrates. If an insecticide selects specific isoenzymes, which can act on different insecticides, cross-resistance might be possible. Maybe the significant correlation between abamectin and methomyl is that methomyl has specific isoenzymes that associated with the abamectin. Resistance to newer chemistry insecticides in $S$. litura has not yet been reported from cash crops growing areas of Hunan, China to the best of our knowledge, except one reported paper in which they have identified resistance in S. litura from two locations in Jiangsu and Anhui Provinces (Huang et al. 2006). Insecticide resistance is an increasing concern in agricultural crops of China against almost all the major insect pests such as cotton bollworm Helicoverpa armigera $(\mathrm{H}).(\mathrm{Wu}$ et al. 2005; Wu 2007), sweet potato whitefly Bemisia tabaci (Gennadius) (Luo et al. 2010; Wang et al. 2010a, b), diamondback moth Plutella xylostella (L.) (Zhao et al. 2006; Wang et al. 2010a, b), western flower thrips Frankliniella occidentalis (Pergande) (Chen et al. 2011), and beet armyworm S. exigua (H.) (Mu et al. 2005; Zhou et al. 2011). These insects have been reported to develop resistance either against different groups or the representative of some group of insecticides. On the other hand, 
illiteracy can be one of the reasons for indiscriminate insecticides use for the development of insecticidal resistance in the most of major pests of cash crops.

Spodoptera litura has recently emerged as a serious pest of cash crops in Hunan, China. The development of a broad-spectrum resistance to insecticides has complicated its chemical control. However, the control of S. litura has relied mainly on the application of various insecticides. It is very important to select several effective insecticides to control this pest. The successful management of insecticide resistance depends ultimately on a thorough knowledge of its genetic basis and the mechanisms involved. The mode of inheritance helps in resistance detection, monitoring, modeling and risk assessment. Such knowledge can provide the basis for management programs aimed at minimizing the development of resistance. From the results of this article, we propose newer and conventional insecticides, which have different resistance mechanisms as effective insecticides rotation program for $S$. litura in Hunan. In order to protect those insecticides and postpone the development of resistance, a resistance management strategy of decreased selection pressure could be achieved by alternations these insecticides on basis of proper pest scouting and pest status for decision of control application or using insecticides when economic injury levels are achieved. Alternative pest management practices, such as cultural, pheromones traps, parasitoids, and predators could also help to reduce the selection pressure. Prognosis on the basis of light or pheromone-traps and prevailing meteorological conditions may help in determining better timing of control operations. Slow-release pheromone formulations have shown success for mating disruption (Wei and Du 2004). It could also help to conserve the parasitoids of $S$. litura or microbial parasites such as nucleopolyhedrovirus (Nathan and Kalaivani 2005; Nguyen et al. 2005), which is necessary to reduce pesticide applications. Bacillus thuringiensis toxins (Cry1Ca and $\mathrm{Cry} 1 \mathrm{~F}$ ) which are also effective against $S$. litura (Zhang et al. 2006) and other major insect pests such as $H$. armigera (Wan et al. 2005; $\mathrm{Wu}$ et al. 2008), stacking them in a crop plant and using as an integrated pest management tool could also be another promising management strategy.

Acknowledgments This research was financially supported by the Special Agricultural Research Projects for Public Welfare, China (201203038), the National Natural Science Foundation of China (31071716), and the Program for New Century Excellent Talents in University (NCET-10-0163).

Conflict of interest The authors have declared that no conflict of interest exists.

Open Access This article is distributed under the terms of the Creative Commons Attribution License which permits any use, distribution, and reproduction in any medium, provided the original author(s) and the source are credited.

\section{References}

Abbott WS (1925) A method of computing the effectiveness of an insecticide. J Econ Entomol 18:265-267

Ahmad M, Arif MI (2010) Resistance of beet armyworm Spodoptera exigua (Lepidoptera: Noctuidae) to endosulfan, organophosphorus and pyrethroid insecticides in Pakistan. Crop Prot 29:1428-1433

Ahmad M, Arif MI, Ahmad M (2007a) Occurrence of insecticide resistance in field populations of Spodoptera litura (Lepidoptera: Noctuidae) in Pakistan. Crop Prot 26:809-817

Ahmad M, Sayyed AH, Crickmore N, Saleem MA (2007b) Genetics and mechanism of resistance to deltamethrin in a field population of Spodoptera litura (Lepidoptera: Noctuidae). Pest Manag Sci 63:1002-1010

Ahmad M, Sayyed AH, Saleem MA (2008) Evidence for field evolved resistance to newer insecticides in Spodoptera litura (Lepidoptera: Noctuidae) from Pakistan. Crop Prot 27: 1367-1372

Armes NJ, Wightman JA, Jadhav DR, Ranga Rao GV (1997) Status of insecticide resistance in Spodoptera litura in Andhra Pradesh, India. Pestic Sci 50:240-248

Bisset J, Rodriguez M, Soca A, Pasteur N, Raymond M (1997) Crossresistance to pyrethroid and organophosphorus insecticides in the southern house mosquito (Diptera: Culicidae) from Cuba. J Med Entomol 34:244-246

Chen Q, Jin QA, Peng ZQ, Tang C, Wen HB (2008) Analysis of the susceptibility of Spodoptera litura (Fabricius) to abamectin. Chin Agric Sci Bull 24:361-364

Chen XL, Yuan LZ, Du YZ, Zhang YJ, Wang JJ (2011) Crossresistance and biochemical mechanisms of abamectin resistance in the western flower thrips, Frankliniella occidentalis. Pestic Biochem Physiol 101:34-38

Delorme R, Fournier D, Chaufaux J, Cuany A, Bride JM, Auge D, Berge JB (1988) Esterase metabolism and reduced penetration are causes of resistance to deltamethrin in Spodoptera exigua HUB (Noctuidea; lepidoptera). Pestic Biochem Phys 32:240-246

Huang SJ, Xu JF, Han ZJ (2006) Baseline toxicity data of insecticides against the common cutworm Spodoptera litura (Fabricius) and a comparison of resistance monitoring methods. Int J Pest Manag 52:209-213

Ishaaya I, Casida JE (1980) Properties and toxicological significance of esterases hydrolyzing permethrin and cypermethrin in Trichoplusia ni larval gut and integument. Pestic Biochem Physiol 14:178-184

Ishtiaq M, Saleem MA, Razaq M (2012) Monitoring of resistance in Spodoptera exigua (Lepidoptera: Noctuidae) from four districts of the Southern Punjab, Pakistan to four conventional and six new chemistry insecticides. Crop Prot 33:13-20

Kranthi KR, Jadhav DR, Wanjari RR, Ali SS, Russell D (2001) Carbamate and organophosphate resistance in cotton pests in India, 1995 to 1999. Bull Entomol Res 91:37-46

Kranthi KR, Jadhav DR, Kranthi S, Wanjari RR, Ali SS, Russell DA (2002) Insecticide resistance in five major insect pests of cotton in India. Crop Prot 21:449-460

LeOra S (2003) Poloplus, a user's guide to probit and logit analysis. LeOra Software, Berkeley

Litchfield JT, Wilcoxon FA (1949) A simplified method of evaluating dose-effect experiments. J Pharmacol Exp Ther 99:99-103 
Luo C, Jones CM, Devine G, Zhang F, Denholm I, Goman K (2010) Insecticide resistance in Bemisia tabaci biotype Q (Hemiptera: Aleyrodidae) from China. Crop Prot 29:429-434

Matsuura H, Naito A (1997) Studies on the cold-hardiness and overwintering of Spodoptera litura F. (Lepidoptera: Noctuidae): VI. Possible overwintering areas predicted from meteorological data in Japan. Appl Entomol Zool 32:167-177

$\mathrm{Mu}$ W, Wu KM, Zhang WJ, Guo YY (2005) Cross-resistance and relative fitness of lambda-cyhalothrin resistant near-isogenic lines in Spodoptera exigua (Hübner). Sci Agric Sin 38:2007-2013

Nathan SS, Kalaivani K (2005) Efficacy of nucleopolyhedrovirus and azadirachtin on Spodoptera litura Fabricius (Lepidoptera: Noctuidae). Biol Control 34:93-98

Nguyen DH, Nakai M, Takatsuka J, Okuno S, Ishii T, Kunimi Y (2005) Interaction between a nucleopolyhedrovirus and the braconid parasitoid Meteorus pulchricornis (Hymenoptera: Braconidae) in the larvae of Spodoptera litura (Lepidoptera: Noctuidae). Appl Entomol Zool 40:325-334

Pérez CJ, Alvarado P, Narváez C, Miranda F, Hernández L, Vanegas H, Hruska A, Shelton AM (2000) Assessment of insecticide resistance in five insect pests attacking field and vegetable crops in Nicaragua. J Econ Entomol 93:1779-1787

Raymond M, Marquine M (1994) Evolution of insecticide resistance in Culex pipiens populations: the Corsican paradox. J Evol Biol 7:315-337

Sahayaraj K, Paulraj MG (1998) Screening the relative toxicity of some plant extracts to Spodoptera litura Fab. (Insecta: Lepidoptera: Noctuidae) of groundnut. Fresenius Environ Bull 7:557-560

Saleem MA, Ahmad M, Aslam M, Sayyed AH (2008) Resistance to selected organochlorin, organophosphate, carbamate and pyrethroid, in Spodoptera litura (Lepidoptera: Noctuidae) from Pakistan. J Econ Entomol 101:1667-1675

Sayyed AH, Haward R, Herrero S, Ferre J, Wright DJ (2000) Genetic and biochemical approach for characterization of resistance to Bacillus thuringiensis toxin Cry1 Ac in a field population of the diamondback moth, Plutella xylostella. Appl Environ Microbiol 66:1509-1516

Sayyed AH, Saeed S, Noor-ul-ane M, Crickmore N (2008) Genetic, biochemical, and physiological characterization of spinosad resistance in Plutella xylostella (Lepidoptera: Plutellidae). J Econ Entomol 101:1658-1666

Scott JG (1999) Cytochromes P450 and insecticide resistance. Insect Biochem Mol Biol 29:757-777

Shad SA, Sayyed AH, Fazal S, Saleem MA, Zaka SM, Ali M (2012) Field evolved resistance to carbamates, organophosphates, pyrethroids, and new chemistry insecticides in Spodoptera litura Fab. (Lepidoptera: Noctuidae). J Pest Sci 85:153-162

Su JY, Lai TC, Li J (2012) Susceptibility of field populations of Spodoptera litura (Fabricius) (Lepidoptera: Noctuidae) in China to chlorantraniliprole and the activities of detoxification enzymes. Crop Prot 42:217-222

Teran-Vargas AP, Garza-Urbina E, Blanco-Montero CA, PerezCarmona G, Pellegaud-Rabago JM (1997) Efficacy of new insecticides to control beet armyworm in north eastern Mexico. In: Proceedings of the Beltwide Cotton Conference of the National Cotton Council, New Orleans, Louisiana, pp. 1030-1031

Torres-Vila LM, Rodriguez-Molina MC, Lacasa-Plasencia A, BielzaLino P (2002) Insecticide resistance of Helicoverpa armigera to endosulfan, carbamates and organophosphates: the Spanish case. Crop Prot 21:1003-1013

Van Leeuwen TV, Pottelberge SV, Tirry L (2006) Biochemical analysis of a chlorfenapyr-selected resistant strain of Tetranychus urticae Koch. Pest Manag Sci 62:425-433

Wan P, Zhang YJ, Wu KM, Huang MS (2005) Seasonal expression profiles of insecticidal protein and control efficacy against Helicoverpa armigera for Bt cotton in the Yangtze River Valley of China. J Econ Entomol 98:195-201

Wang JJ, Dong HG, Yuan LZ (2009) Resistance mechanisms of Spodoptera litura to indoxacarb. Acta Phytophysiol Sin 36:168-172

Wang XL, Li XY, Shen AD, Wu YD (2010a) Baseline Susceptibility of the diamondback moth (Lepidoptera: Plutellidae) to chlorantraniliprole in China. J Econ Entomol 103:843-848

Wang ZY, Yan HF, Yang YH, Wu YD (2010b) Biotype and insecticide resistance status of the whitefly Bemisia tabaci from China. Pest Manag Sci 66:1360-1366

Wei HY, Du JW (2004) Sublethal effects of larval treatment with deltamethrin on moth sex pheromone communication system of the Asian corn borer, Ostrinia furnacalis. Pestic Biochem Physiol 80:12-20

Wu KM (2007) Monitoring and management strategy for Helicoverpa armigera resistance to Bt cotton in China. J Invertebr Pathol 95:220-223

Wu SC, Gu YZ, Wang DS (1995) Resistance of the tobacco army moth (Prodenia litura) to insecticides and its control. Acta Agric Shanghai 11:39-43

Wu KM, Mu W, Liang GM, Guo YY (2005) Regional reversion of insecticide resistance in Helicoverpa armigera (Lepidoptera: Noctuidae) is associated with the use of Bt cotton in northern China. Pest Manag Sci 61:491-498

Wu KM, Lu YH, Feng HQ, Jiang YY, Zhao JZ (2008) Suppression of cotton bollworm in multiple crops in China in areas with Bt toxin-containing cotton. Science 321:1676-1678

Xie SH, Liang YP, Lin ZF, Li H, Ji XC (2010) The toxicity and control efficiency of 9 insecticides to Spodoptera litura. Plant Prot 36:175-177

Zhang GF, Wan FH, Liu WX, Guo JY (2006) Early instar response to plant-delivered Bt-toxin in a herbivore (Spodoptera litura) and a predator (Propylaea japonica). Crop Prot 25:527-533

Zhao JZ, Collins HL, Li YX, Mau RFL, Thompson GD, Hertlein M, Andaloro JT, Boykin R, Shelton AM (2006) Monitoring of diamondback moth (Lepidoptera: Plutellidae) resistance to spinosad, indoxacarb, and emamectin benzoate. J Econ Entomol 99:176-181

Zhou C, Liu YQ, Yu WL, Deng ZR, Gao M, Liu F, Mu W (2011) Resistance of Spodoptera exigua to ten insecticides in Shandong, China. Phytoparasitica 39:315-324 